\title{
Traitement à la chaux des tufs volcaniques du Sénégal oriental \\ Activation de la réaction pouzzolanique par du phosphogypse
}

\author{
Mouhamadou Bassir Diop', Abdoulaye Boiro', Raoul Jauberthie ${ }^{3}$, Ahmed Bouguerra $^{4}$ \\ I. Institut des Sciences de la Terre (I.S.T.), BP 5396, Faculté des sciences et techniques, Université Cheikh Anta Diop de Dakar, Sénégal \\ 2. Département de géologie, BP 5385, Faculté des Sciences et Techniques, Université Cheikh Anta Diop de Dakar, Sénégal \\ 3. Laboratoire de génie civil et génie mécanique - INSA - 20, avenue des Buttes de Coësmes, CS I43I5 - 35043 Rennes Cedex - France \\ 4. 31, rue Mirabeau - Résidence Edgar-Degas - 35000 Rennes - France
}

Pour toute correspondance : mbdiop@ucad.sn ou bassir@geomat-afrique.com

\section{Résumé}

\begin{abstract}
Au Sénégal, des déchets appelés phosphogypse [I] résultent de la fabrication de l'acide phosphorique à partir du phosphate. Ils sont disposés en tas dont la qualité et la quantité restent non spécifiées. L'évaluation du stock de phosphogypse montre une quantité disponible estimée à 166 millions de $\mathrm{m}^{3}$. Au point de vue environnemental, le lessivage de ces tas par les eaux de pluie pendant la saison des pluies rend les sols avoisinants acides.

La région de Mako (Sénégal oriental) est pourvue d'une quantité importante de tufs volcaniques tendres et pulvérulents provenant du volcanisme calco-alcalin de l'âge birimien (environ 2 milliards d'années). Ces tufs volcaniques sont acides (avec près de $70 \%$ de $\mathrm{SiO}_{2}$ ). Cette forte teneur en silice et en aluminium (13\%) confère aux mélanges tufs + liant des propriétés pouzzolaniques [2]. Traités à la chaux (2,5\%), les tufs permettent d'obtenir des briques de résistances élevées (3 $\mathrm{MPa}$ ) dès 72 heures de conservation à $80{ }^{\circ} \mathrm{C}$. L'apport de phosphogypse accélère considérablement la réaction pouzzolanique et induit un gain de résistance de près de $60 \%$ permettant l'utilisation des tufs en géotechnique routière et pour la construction.
\end{abstract}

Mots clés : tufs volcaniques, activité pouzzolanique, traitement, chaux, route, phosphogypse, habitat.

\section{Introduction}

Les tufs volcaniques tendres d'âge birimien (environ 2 milliards d'années) de Mako sont des pyroclastites très anciennes altérées et dévitrifiées [3]. Ils forment de larges affleurements au Sénégal oriental et présentent des réserves considérables. Ils constituent l'essentiel des sols de fondation dans cette région. Ils présentent des caractéristiques géotechniques médiocres. Grâce à leur finesse et leur tendresse, ces matériaux se prêtent bien à un traitement aux liants. Le travail présenté ici fait le point sur le traitement à la chaux vive $(\mathrm{CaO})$ de ces tufs et l'incidence de l'ajout de phosphogypse sur la réaction pouzzolanique.

\section{Matériaux et méthodes d'étude}

Les tufs utilisés proviennent du Sénégal oriental (Bafoundou). Du fait leur caractère tendre, ils sont facilement pulvérisés au broyeur à mâchoire. Ensuite, le refus à $250 \mu \mathrm{m}$ est repris au broyeur à anneau. C'est le tamisat à $250 \mu \mathrm{m}$ qui a été utilisé dans les formulations.

L'analyse chimique des tufs est donnée par le tableau I. C'est un tuf acide $\left(\mathrm{SiO}_{2}>66 \%\right)$, riche en fer et en alumine. La différence entre la teneur en silice et en chaux supérieure à $34 \%$ traduit une grande fraction vitreuse dans les tufs.

Pour déterminer approximativement l'amplitude des teneurs en chaux à tester, nous avons utilisé la méthode proposée par Pichon [4]. Cette méthode est basée sur la détermination du potentiel de chaux combiné (PCC). C'est la quantité optimale de chaux combinée par unité de masse du matériau.

Concernant les propriétés physiques, elles sont résumées dans le tableau 2. La densité des tufs est supérieure à celle de la silice (2.65) à cause de sa richesse en fer. C'est un matériau qui possède une surface spécifique considérable qui est pratiquement le double de celle des ciments portland traditionnels. Cette finesse (figure I) du matériau est confirmée par la granulométrie au laser qui donne un grain moyen aux environs de $16 \mu \mathrm{m}$. D'après le diagramme

Tableau I : Composition chimique des tufs utilisés et phase réactive

\begin{tabular}{|c|c|c|c|c|c|c|c|c|c|c|}
\hline $\mathrm{SiO}_{2}$ & $\mathrm{Al}_{2} \mathrm{O}_{3}$ & $\mathrm{Fe}_{2} \mathrm{O}_{3}$ & $\mathrm{CaO}$ & $\mathrm{MgO}$ & $\mathrm{K}_{2} \mathrm{O}$ & $\mathrm{Na}_{2} \mathrm{O}$ & $\mathrm{TiO}_{2}$ & $\mathrm{Mn}_{2} \mathrm{O}_{3}$ & $\mathrm{PF}$ & Silice réactive \\
\hline 69.7 & 13.3 & 10.1 & 0.1 & 0.1 & 1.3 & 0.6 & 0.8 & 0.1 & 4 & 8.9 \\
\hline
\end{tabular}


Tableau 2 : Paramètres physico-chimiques et géotechniques des tufs

\begin{tabular}{|c|c|c|c|c|c|c|c|c|c|}
\hline $\begin{array}{c}\text { Chapelle } \\
(\mathrm{mg} / \mathrm{g})\end{array}$ & $\begin{array}{c}\text { SSB } \\
\text { chapelle } \\
\left(\mathrm{m}^{2} / \mathrm{g}\right)\end{array}$ & $\begin{array}{c}\text { BET } \\
(\mathrm{ground}) \\
\left(\mathrm{m}^{2} / \mathrm{g}\right)\end{array}$ & $\begin{array}{c}\text { Densité } \\
\left(\mathrm{g} / \mathrm{cm}^{3}\right)\end{array}$ & $\begin{array}{c}\text { SSB } \\
\left(\mathrm{m}^{2} / \mathrm{g}\right)\end{array}$ & $\begin{array}{c}\text { PCC } \\
(\mathrm{g} / \mathrm{g})\end{array}$ & $\begin{array}{c}\mathrm{w}_{\mathrm{L}} \\
(\%)\end{array}$ & $\begin{array}{c}\mathrm{W}_{\mathrm{P}} \\
(\%)\end{array}$ & \multicolumn{2}{|c|}{$\begin{array}{c}\text { Optimum Proct } \\
\mathrm{w}(\%) \rho\left(\mathrm{g} / \mathrm{cm}^{3}\right)\end{array}$} \\
\hline 162 & 9924 & 8.37 & 2.82 & 7111 & 0,53 & 43 & 30 & 15,3 & 1,8 \\
\hline
\end{tabular}

Tableau 3 : Caractéristiques physico-chimiques de la chaux

\begin{tabular}{|c|c|c|c|c|}
\hline $\begin{array}{c}\text { Surface spécifique Blaine } \\
\left(\mathrm{cm}^{2} / \mathrm{g}\right)\end{array}$ & $\begin{array}{c}\text { Masse } \\
\text { volumique } \\
\left(\mathrm{kg} / \mathrm{m}^{3}\right)\end{array}$ & $\begin{array}{c}\text { Poids spécifique } \\
\left(\text { Tonne } / \mathrm{m}^{3}\right)\end{array}$ & $\begin{array}{c}\text { Indice } \\
\text { d'hydraulicité }\end{array}$ & $\begin{array}{c}\text { Type de prise } \\
\text { aérienne }(\%)\end{array}$ \\
\hline 8.000 à 20.000 & 490 à 700 & 2.2 à 2.5 & 0 à 0,1 & 100 \\
\hline
\end{tabular}

Tableau 4 : Composition chimique moyenne du phosphogypse

\begin{tabular}{|l|l|l|l|l|l|}
\hline Éléments majeurs & $\mathrm{SO}_{3}$ & $\mathrm{CaO}$ & $\mathrm{SiO}_{2}$ & $\mathrm{P}_{2} \mathrm{O}_{5}$ & $\mathrm{PF}$ \\
\hline Pourcentage & 42.3 & 30.3 & 4.2 & 0.5 & 21.1 \\
\hline
\end{tabular}

de Casagrande, les tufs peuvent être classés parmi les limons peu plastiques.

Figure I : Granulométrie au laser des tufs

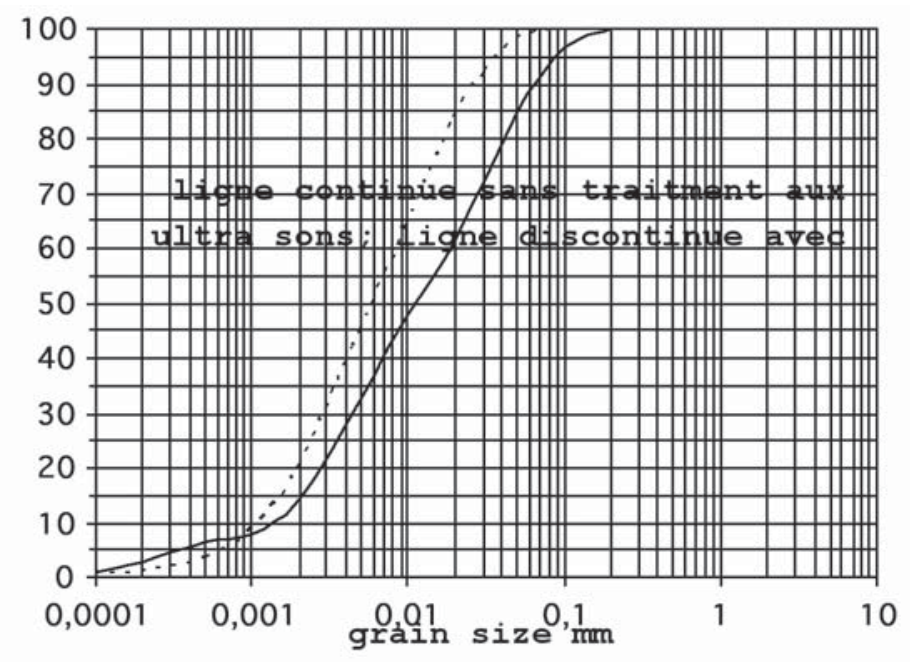

Selon [5], l'activité pouzzolanique potentielle d'un matériau réside dans une teneur en silice supérieure à $45 \%$, une proportion importante de phase vitreuse et une grande surface spécifique. Ces caractéristiques chimiques, minéralogiques et physiques sont retrouvées dans les tufs volcaniques étudiés, même si la phase vitreuse est dévitrifiée, à cause de l'altération qu'ils ont subie.

La chaux utilisée dans la stabilisation des tufs est une chaux vive (CaO). Ses caractéristiques sont mentionnées sur le tableau 3. Le processus de fabrication de l'acide phosphorique au niveau des Industries Chimiques du Sénégal (I.C.S) entraîne la formation de résidu riche en gypse qui, après lavage et essorage, donne le phosphogypse. L'évaluation du stock de phosphogypse montre une quantité disponible estimée à 166 millions de $\mathrm{m}^{3}$.
Tableau 5 : Composition des formules

\begin{tabular}{||l|c|c|c|c||}
\hline$N^{\circ}$ des formules & 1 & 2 & 3 & 4 \\
\hline Tufs (\%) & 100 & 96 & 96 & 94 \\
\hline $\mathrm{CaO}(\%)$ & & 4 & 4 & 4 \\
\hline Phosphogypse (\%) & & & & 2 \\
\hline
\end{tabular}

L'analyse chimique du phosphogypse est consignée dans le tableau 4. Le phosphogypse présente une granulométrie se situant entre $0 \mu \mathrm{m}$ et $100 \mu \mathrm{m}$, avec $30 \%$ de grains inférieurs à $40 \mu \mathrm{m}$.

Pour les tests de fabrication, quatre formulations à base de tufs et de chaux ont été étudiées. Le phosphogypse qui est un activant est utilisé dans la formule 4. Les compositions de ces formules sont décrites dans le tableau 5. Selon [6], l'addition de I à $3 \%$ de gypse au ciment pouzzolane chaux peut avoir parfois une conséquence bénéfique sur le développement des résistances mécaniques mais cette effet est aléatoire et reste imprévisible.

Pour estimer les possibilités d'application en géotechnique routière, nous avons utilisé les essais Proctor et CBR (Californian Bearing Ratio). Les pâtes des formules 2, 3 et 4, conditionnées dans des sacs en plastiques hermétiquement fermés, ont subi une maturation pendant 48 heures avant leur compactage. Le tableau 6 donne les normes de résistance pour les briques de terre comprimée (BTC) au Sénégal.

Les essais sur briquettes ont pour but de déterminer la formulation idéale. Trois paramètres doivent être optimisés : la teneur en chaux, la teneur en eau et la température. Toutefois, ayant constaté dans les essais préliminaires un important gonflement des éprouvettes dû sans doute à la présence d'argiles gonflantes telles que l'illite (révélée par la diffractométrie des $R X$ effectuée sur les tufs), toutes les éprouvettes sont enveloppées dans du papier en aluminium de façon à les isoler de l'humidité ambiante. Les éprouvettes cylindriques ont un diamètre de $2 \mathrm{~cm}$ sur une hauteur de $4 \mathrm{~cm}$.

L'optimisation de l'eau de gâchage a consisté à faire varier la teneur en eau entre 30 et $60 \%$ en poids des matières solides (tufs et chaux). En dessous de $30 \%$ d'eau, le 
Tableau 6 : Caractéristique des BTC au Sénégal ( : structure porteuse, 2 : structure non porteuse)

\begin{tabular}{|c|c|c|c|}
\hline Catégorie & $\begin{array}{c}\text { Résistance à la compression } \\
\text { sèche }(\mathrm{MPa})\end{array}$ & $\begin{array}{c}\text { Résistance à la compression } \\
\text { humide }(\mathrm{MPa})\end{array}$ & $\begin{array}{c}\text { Absorption d'eau } \\
(\%)\end{array}$ \\
\hline 1 & $\geq 2$ & $\geq 1$ & \multirow{2}{*}{15} \\
\hline 2 & $\geq 4$ & $\geq 2$ & 15 \\
\hline
\end{tabular}

Tableau 7 : Formules utilisées dans l'étude (\% en poids)

\begin{tabular}{|c|c|c|c|c|c|c|c|c|c|c|}
\hline Formules & 40 & 41 & 42 & 43 & 44 & 70 & 72 & 74 & 76 & 78 \\
\hline Chaux (\%) & 4 & 4 & 4 & 4 & 4 & 7 & 7 & 7 & 7 & 7 \\
\hline Phosphogypse (\%) & 0 & 1 & 2 & 3 & 4 & 0 & 2 & 4 & 6 & 8 \\
\hline Tufs (\%) & 96 & 95 & 94 & 93 & 92 & 93 & 91 & 89 & 87 & 85 \\
\hline
\end{tabular}

mélange est trop maigre (impossible à travailler). Au dessous de $60 \%$, il est trop fluide. L'optimisation de la teneur en chaux consiste à faire varier la teneur en chaux entre I et $20 \%$ de chaux en poids des matières solides. La température active la réaction pouzzolanique [7]. L'optimisation de la température de maturation consiste à faire varier la température du milieu de conservation entre $20{ }^{\circ} \mathrm{C}$ et $120^{\circ} \mathrm{C}$.

Pour les essais sur briques, le principe des formulations consiste à utiliser le minimum de chaux et de phosphogypse tout en ayant des briques ayant des caractéristiques conformes à la norme sénégalaise. Les formules testées sont consignées dans le tableau 7.

Le mélange se fait d'abord à sec entre les différents matériaux. Le malaxage doit être bien soigné. Le mélange est contrôlé en appréciant l'unité de la teinte : aucune traînée de chaux non incorporée dans le mélange ne doit apparaître. La quantité d'eau ajoutée est fixée à 1500 g, soit $30 \%$. Le tout est ensuite bien mélangé jusqu'à l'obtention d'une pâte très homogène. Cependant, si la teneur en eau de mouillage est trop élevée (supérieure à $30 \%$ ), la pression de l'engin de compactage est amortie par l'eau qui ne peut être chassée d'entre les grains. Ainsi, la presse ne pourra pas permettre la confection des briques. Les éprouvettes sont confectionnées à l'aide d'une presse Terstaram munie d'un moule de $22 \mathrm{~cm}$ de côté exerçant une pression nominale égale à $21 \mathrm{~kg} / \mathrm{cm}^{2}$. Ce moule a été ensuite divisé en deux parties égales. Ce qui permet d'obtenir deux éprouvettes de dimensions: $22 \times 1 \mid \times 5,5 \mathrm{~cm}$.

\section{Résultats des essais}

Pour les essais sur briquettes, le graphique de la figure 2 fait ressortir une courbe en cloche asymétrique à pente raide avec un optimum à $50 \%$ pour la teneur en eau. II est intéressant de constater que l'optimum de teneur en eau correspondant à la densité maximum des éprouvettes est différent ; il est de $40 \%$.

L'optimum de chaux (7 \%) est supérieur à la valeur généralement trouvée pour les argiles [8] qui est d'environ $4 \%$ avec la chaux vive $(\mathrm{CaO})$ et $5 \%$ avec la chaux éteinte $\left(\mathrm{Ca}(\mathrm{OH})_{2}\right)$. Cette différence est en rapport avec I'extrême finesse des tufs et leur réactivité. Ici, il est important
Figure 2 : Optimisation des paramètres de maturation
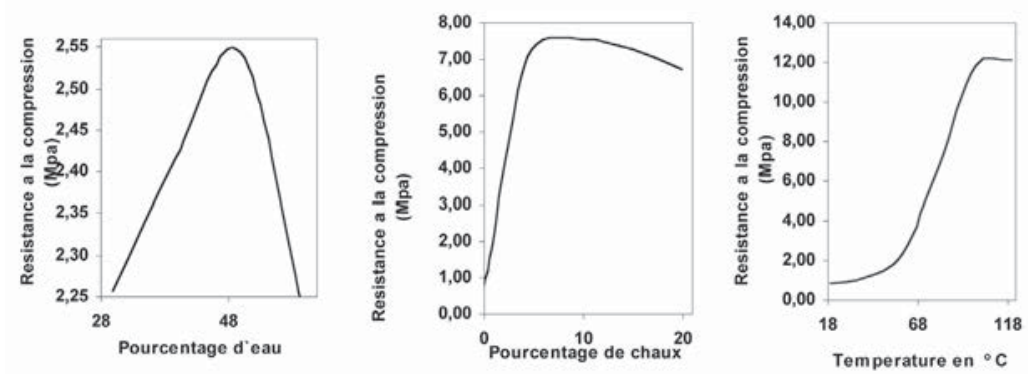

de noter le caractère pouzzolanique des tufs qui, dans les conditions d'essai, présentent une résistance à la compression de $5.3 \mathrm{MPa}$ dès trois jours de conservation. La réaction pouzzolanique démarre véritablement aux environs de $57^{\circ} \mathrm{C}$. Mais, elle ne devient véritablement effective qu'à $70{ }^{\circ} \mathrm{C}$. Enfin, à $100{ }^{\circ} \mathrm{C}$, débute un palier qui semble se maintenir au delà de $120^{\circ} \mathrm{C}$. $\|$ est donc inutile de conserver les éprouvettes au delà de $100^{\circ} \mathrm{C}$.

L'étude de diffraction de rayons $X$ (figure 3 ) montre dans ces tufs volcaniques altérés et dévitrifiés la paragénèse minérale suivante: quartz-hématite-kaolinite-illite-silice amorphe. L'amorphe ou phase vitreuse se traduit aux rayons $X$ par des dômes de diffusion [4]. Ainsi, d'après l'importance des dômes, la proportion de phase vitreuse dévitrifiée semble importante. La diffractométrie des $R X$ des tufs bruts et des tufs traités à 2, 7, 12, 17 et $22 \%$ de chaux vive $(\mathrm{CaO})$ révèle une nette évolution des phases minérales. Les minéraux argileux sont nettement visibles au microscope électronique à balayage. Après traitement à la chaux, les pics caractéristiques de la kaolinite s'estompent dès l'ajout de $2 \%$ de chaux, preuve que la réaction pouzzolanique est bien effective. Les pics du quartz sont nettement moins visibles (réduction de plus $50 \%$ de l'intensité) et tous les pics sont décalés d'environ $1^{\circ}$. Enfin, les pics caractéristiques de l'hématite ont presque disparu. Par rapport à l'ajout de chaux, la teneur en chaux à $7 \%$ correspond à la réaction pouzzolanique la plus effective. Ce qui confirme les résultats sur briquettes.

Au microscope électronique à balayage, les mousses de silicate de calcium hydraté (CSH) sont bien visibles (figure 4). Les paramètres de maturation des éprouvettes ayant été bien maîtrisés, des essais de longue durée sont exécutés afin de suivre l'évolution de la résistance des éprouvettes dans le temps. Afin de minimiser la quantité de chaux pour 
Figure 3 : Diffractométrie aux $R X$ des tufs bruts et traités

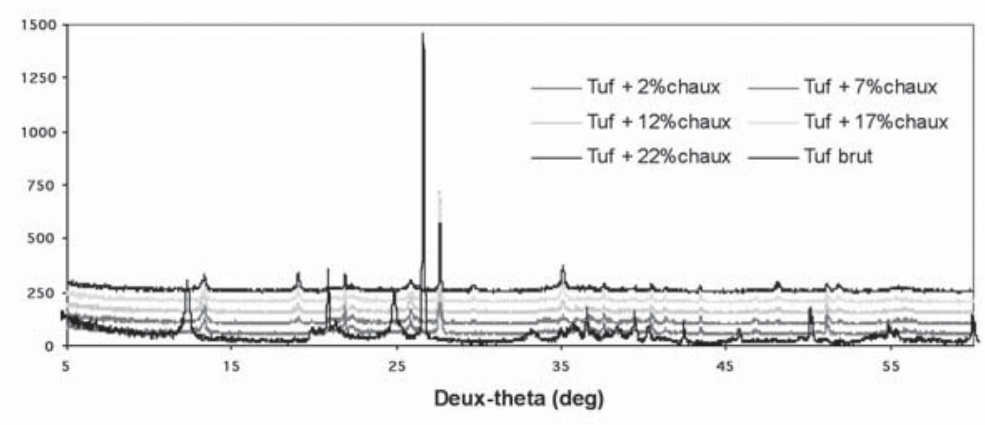

des raisons économiques, nous avons travaillé pour les essais de longue durée avec $2,5 \%$ de chaux. Les éprouvettes sont écrasées après 2, 7, 14, 28 et 90 jours de conservation. La figure 5 confirme le caractère lent de l'expression de l'effet pouzzolanique. Toutefois, les performances obtenues, supérieures à $4 \mathrm{MPa}$, montre qu'il est possible de travailler à de très faibles teneurs en chaux. Les tufs calcinés à $800{ }^{\circ} \mathrm{C}$ sont moins réactifs que les tufs bruts.

Figure 5 : Essai mécanique longue durée

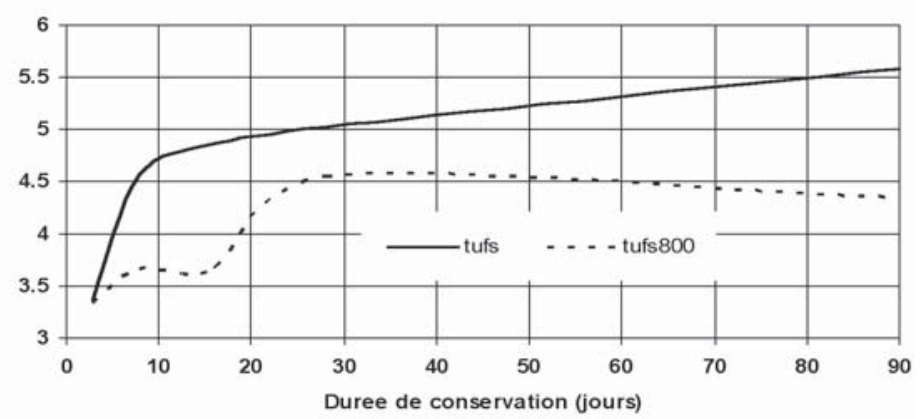

Pour les essais sur briques, la figure 6 correspond aux résistances à la compression sèche des éprouvettes après 7 jours de cure à l'étuve à la température de $60{ }^{\circ} \mathrm{C}$ avec une teneur en chaux fixée à $7 \%$. Les résistances à la compression sèche obtenues sont élevées. D'après la figure, la résistance à la compression sèche croît lorsqu'on augmente progressivement la teneur en phosphogypse. Un palier semble se dessiner entre 2 et $6 \%$ de phosphogypse. Ensuite, la courbe présente une pente ascendante.

Figure $\mathbf{6}$ : Variation de la résistance en fonction de la teneur en phosphogypse à $7 \%$ de chaux à $60^{\circ} \mathrm{C}$

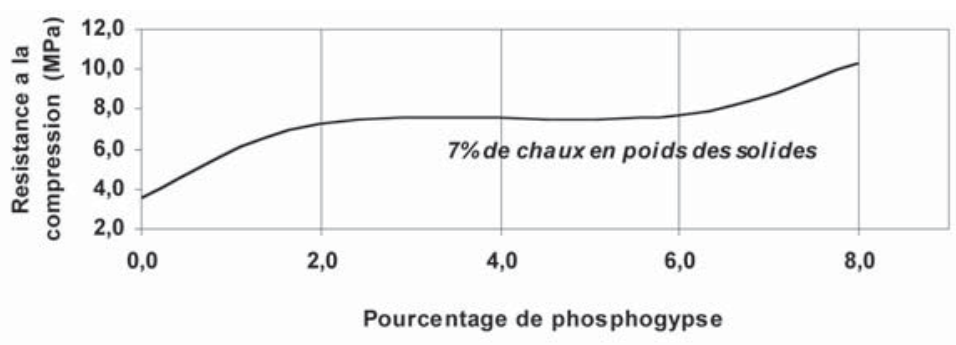

Le tableau 8 présente les résultats des essais de compactage. La valeur du CBR des tufs seuls est faible (8\%) et ne permet son emploi en géotechnique routière qu'en couche de fondation. Avec un traitement à $4 \%$ de chaux, la
Figure 4 : a) MEB des tufs de Mako : des aloysites en choux-fleur, aloysite en tubes, des plaquettes de kaolinite et des grains de quartz ; b) Feutrage de $\mathrm{CSH}$; texture filamenteuse.


portance des tufs est nettement améliorée, le CBR est alors égal à $30 \%$ mais l'utilisation d'un tel matériau se limite aux couches de fondation des assises routières.

Tableau 8 : Valeurs de CBR obtenues avec les formules

\begin{tabular}{|l|c|c|c|c|c|c|}
\cline { 2 - 7 } \multicolumn{1}{c|}{} & \multicolumn{2}{|c|}{ Exigences couches } & \multicolumn{4}{c|}{$\mathrm{N}^{\circ}$ des formules } \\
\cline { 2 - 7 } \multicolumn{1}{c|}{} & Fond & base & 1 & 2 & 3 & 4 \\
\hline CBR $(\%): 3$ jours à l'air et 4 jours d'imbibition & $6,0-80$ & $\geq 160$ & 8 & 30 & & \\
\hline CBR (\%): 7 jours à $60^{\circ} \mathrm{C}$ & $80-120$ & & & & 140 & 195 \\
\hline CBR (\%): 7 jours à $60^{\circ} \mathrm{C}$ et 4 jours d'imbibition & $6,0-80$ & $\geq 160$ & & & 158 & 210 \\
\hline $\begin{array}{l}\text { Résistance à la compression : 3 jours à l'air et } \\
4 \text { jours d'imbibition (bars) }\end{array}$ & $2.5-5,0$ & $\geq 5,0$ & \multicolumn{4}{|c|}{} \\
\hline Résistance à la compression 7 jours à l'air (bars) & $5,0-10$ & $18-30$ \\
\hline
\end{tabular}

Avec la chaux, quand les moules sont chauffés à l'étuve de $60{ }^{\circ} \mathrm{C}$, la valeur du CBR est multipliée par plus de 10 (I40\%). L'ajout de phosphogypse, qui est un activant, crée un gain de 40 \% sur le CBR (195\%). Les tufs traités à la chaux et activés au phosphogypse sont utilisables en couche de base. Pour la formule 4, nous avons procédé à un poinçonnement après 4 jours supplémentaires d'imbibition. Nous constatons une amélioration du CBR de $8 \%$. La formule 4 activée à $2 \%$ de phosphogypse présente un CBR très élevée (210\%).

\section{Interprétation des résultats}

La silice et l'alumine libérées lors de la dissolution des minéraux argileux sous l'effet de la chaux vive réagissent avec le calcium en solution pour former par précipitation des silicates calciques hydratés $(\mathrm{CSH})$ et des aluminates calciques hydratés $(\mathrm{CAH})$. Le rôle des $\mathrm{CAH}$ dans les liants pouzzolanes-chaux est toujours accessoire car la cohésion résultant de l'activité pouzzolanique est principalement due aux CSH peu basiques et riches en eau. En effet, des expériences antérieures $[\mathrm{II}$ ont mis en évidence un rapport Si/Al plus important dans les produits de l'activité pouzzolanique que les matériaux de base.

Au dessous de $40{ }^{\circ} \mathrm{C}$, briques curées à l'air, les résistances à la compression obtenues sont faibles. Ceci s'explique par le fait que la réaction pouzzolanique s'est déroulée partiellement. Pour que celle,ci soit complète, il faut que la température de cure de séchage soit supérieure ou égale à $40{ }^{\circ} \mathrm{C}$. La technique d'activation thermique consiste à augmenter la température de cure de séchage en utilisant 
pour cela une étuve ou une bâche en polyane. La technique d'activation chimique consiste à ajouter du phosphogypse au mélange avant le mouillage. L'adjonction de phosphogypse au mélange présente plusieurs effets, notamment l'augmentation de la résistance mécanique. Ceci confirme les travaux de [6]. L'ajout de phosphogypse occasionne un léger accroissement de la densité.

Par rapport à la sensibilité à l'eau, l'adjonction de phosphogypse ne semble pas améliorer la tenue à l'eau des éprouvettes. Le rapport des résistances sec et humide est constant et égal à 2,2 aussi bien pour la formule avec phosphogypse que celle sans.

Les tufs calcinés à $800^{\circ} \mathrm{C}$ sont nettement moins réactifs que les tufs bruts, quelle que soit l'échéance. La transformation du kaolin en métakaolin (plus réactif) à partir de $550{ }^{\circ} \mathrm{C}$ laisse présager l'inverse. De plus, les travaux antérieurs [10] ont montré que les argiles pouvaient représenter jusqu'à près de $30 \%$ de la composition normative des tufs, et la calcination des matériaux vitreux augmente généralement leur finesse. L'explication du phénomène pourrait résider dans le fait que les hydroxydes de fer se transforment en oxydes moins réactifs.

Enfin, l'ajout de phosphogypse au mélange ne provoque ni la création de minéraux nouveaux, ni un changement de texture des briques. Cependant, l'observation des photographies effectuées au microscope électronique à balayage (MEB) montre la présence de mousses de silicate de calcium hydraté (CSH) bien visibles. On peut en déduire que les cristaux observés à l'oeil nu sont constitués de silicates de calcium hydratés et d'hydroxyde de calcium. L'ettringite et le monosulfo-aluminate pourraient être présents lorsque le sulfate de calcium (phosphogypse) s'ajoute aux réactifs $[\mathrm{I} \mid \mathrm{l}$, mais ces deux minéraux n'ont pas été observés sur nos clichés. Le passage d'une texture vésiculaire à une texture filamenteuse résulte de la réaction pouzzolanique entre la silice, l'alumine et la chaux vive.

Pour les essais de compactage routier, avec le traitement à la chaux, quand les moules sont conditionnés à l'étuve de $60{ }^{\circ} \mathrm{C}$, la valeur du CBR est multipliée par plus de 10 (140\%) du fait de l'activation thermique de la réaction pouzzolanique. L'ajout de phosphogypse qui est un activant crée un gain de 40 \% sur le CBR (195\%). La présence de l'activant (phosphogypse) accélère la réaction pouzzolanique et permet d'atteindre rapidement les valeurs de palier. II est important de noter que ces deux formules à base de chaux après une semaine de traitement thermique résistent parfaitement à l'action de l'eau. A ce moment, la réaction pouzzolanique a pris définitivement le pas sur le gonflement. L'eau devient ainsi un milieu favorable à la cinétique de la réaction pouzzolanique.

\section{Conclusion}

Les tufs volcaniques du Sénégal oriental se prêtent bien à un traitement à la chaux. Le traitement au ciment n'est pas économiquement envisageable. Toutefois, pour les tufs traités à la chaux, il faut éviter leur contact avec l'eau dès les premiers jours de cure pour éviter le gonflement des argiles. Au Sénégal oriental, la température avoisine et dépasse $50{ }^{\circ} \mathrm{C}$ à partir du mois du mars. Ce qui permet une activation thermique naturelle de la réaction pouzzolanique. Toutefois, comme celle-ci est lente, l'utilisation de phosphogypse comme activant chimique permet d'avoir rapidement un matériau conforme aux normes sénégalaises sur les briques de terre comprimée pour la construction et la géotechnique routière. Ceci permet par ailleurs d'éviter la mise en tas de ce phosphogypse et ses conséquences néfastes sur l'environnement. De surcroît, les espaces antérieurement occupés par ces tas peuvent ensuite être réaffectés à d'autres usages, notamment agricoles.

\section{Références :}

[I] Prayon Rupel Technologies (1996) : Section conversion et qualité des sous-produits de sulfate de calcium ex-phosphate de Taïba - Test CPP (Central Prayon Process)

[2] Coatanlem, (2004) : Pouzzolanicité des tufs volcaniques acides du Sénégal oriental, XXIle RUGC 2004, Ville \& Génie civil, 3 \& 4 juin 2004, Marne-la-Vallée.

[3] N'Diaye, Matar et al. (2003) : «Pouzolanic activity of acid and intermediary volcanic tuffs of Mako areas (Senegal) », International symposium on industrial minerals and building stones, September 15-18.

[4] Pichon H. (1992) : Le système « Pouzzolane naturelle chaux 》 à 38 et $100^{\circ} \mathrm{C}$, relation entre la composition chimique, les phases néoformées et les conséquences physico-chimiques, Thèse de doctorat, Université Joseph-Fourrier, Grenoble.

[5] Largent (1975): Estimation de l'activité pouzzolanique. Recherche d'un essai. Bull. Liaison Labo. P. et Ch., 93, janvier-février, p. 61-65.

[6] Lea, F. M. (1974): The chemistry of cement and concrete, $3^{\text {rd }}$ Edition, Edward Arnold, New York.

[7] Caijun Shi, (200I) : An overview on the activation of reactivity of naturals, Can. J. Eng, 28, 778-786.

[8] Perret P, (1977) : Contribution à l'étude de la stabilisation des sols fins par la chaux : étude globale du phénomène et applications. Thèse de Doct. Ing, INSA de Rennes, 166 p.

[9] Fournier M. et Geoffray J. M., (1978) : Le liant pouzzolanes-chaux. Bulletin de liaison du laboratoire des Ponts et Chaussés, $n^{\circ}$ 93, réf. 2145, LCPC.

[10] Le Borgne T., Meyer S. (2005) : Pouzzolanicite de tufs volcaniques; fabrication de blocs de construction pour l'habitat traditionnel ; Master Pro aménagement, Université Blaise-Pascal, Clermont-Ferrand.

[I I] Desgache G., Piquel J. (2003) : Etude de cas : Mise en évidence de l'activité pouzzolanique de deux matériaux volcaniques du massif central, DESS de géologie de l'aménagement, Université Blaise-Pascal de Clermont-Ferrand, UFR Sciences exactes et naturelles, 5 I p. 